\title{
Enolase 1 differentially contributes to cell transformation in lung cancer but not in esophageal cancer
}

\author{
JO-MEI MAUREEN CHEN ${ }^{1 *}$, SHAO-CHIH CHIU ${ }^{2,3 *}$, KUN-CHIEH CHEN $^{4,5 *}$, \\ YUN-RU JAOYING HUANG ${ }^{1}$, YU-TING AMBER LIAO ${ }^{1,2}$ and CHANG-TZE RICKY YU ${ }^{1}$ \\ ${ }^{1}$ Department of Applied Chemistry, National Chi Nan University, Puli, Nantou 54561; ${ }^{2}$ Center for Neuropsychiatry, \\ China Medical University Hospital, Taichung 40447; ${ }^{3}$ Graduate Institute of Immunology, China Medical University, \\ Taichung 40402; ${ }^{4}$ Division of Chest Medicine, Department of Internal Medicine, Taichung Veterans General Hospital, \\ Taichung 40705; ${ }^{5}$ Department of Life Sciences, National Chung Hsing University, Taichung 402, Taiwan, R.O.C.
}

Received January 30, 2019; Accepted December 20, 2019

DOI: 10.3892/ol.2020.11427

\begin{abstract}
Enolase transforms 2-phospho-D-glycerate into phosphoenolpyruvate during glycolysis. The human enolase (ENO) family comprises three members named ENO3, which is restricted to muscle tissues, ENO2, which is neuron- and neuroendocrine tissue-specific, and ENO1, which is expressed in almost all tissues. ENO1 is involved in various types of human cancer, including retinoblastoma, hepatocellular carcinoma, pancreatic cancer, renal cell carcinoma, cholangiocarcinoma and gastric cancer. Furthermore, ENO1 enhances cell transformation in numerous cancer cell lines. It has been reported that ENO1 is involved in various activities that are detrimental to cell transformation, including apoptosis and differentiation. However, a few studies demonstrated that ENO1 can be downor upregulated in various types of lung cancer, which suggests that ENO1 has an ambiguous role in the development of lung cancer. The present study aimed to investigate the differential influences of ENO1 on various types of cancer, and to clarify the role of ENO1 in lung cancer in particular. Western blotting was performed to assess ENO1 protein expression levels in lung cancer and esophageal cancer tissues. Furthermore, exogenous ENO1 was overexpressed in cell lines derived from various tissues and single cell proliferation, flowcytometric analysis, and western blotting were performed to determine the cell proliferation rate, cell transformation status, cell cycle progression and the expression of cell cycle regulators, such as cyclins and cyclin-dependent kinases, and survival factors, such as MAPK and AKT. The results demonstrated that ENO1 was upregulated
\end{abstract}

Correspondence to: Professor Chang-Tze Ricky Yu, Department of Applied Chemistry, National Chi Nan University, 1 University Road, Puli, Nantou 54561, Taiwan, R.O.C.

E-mail: ctyu@ncnu.edu.tw

*Contributed equally

Key words: enolase 1, lung cancer, esophageal cancer, cyclin-dependent kinase 6,p38, protein kinase B in collected panels of lung cancer tissues, but not in esophageal cancer tissues. In addition, overexpression of ectopic ENO1 promoted cell proliferation and survival in lung cancer cell lines, which was not the case in other cells, including an esophageal cell line. Furthermore, mechanistic analyses revealed that ENO1 enhanced cell proliferation by accelerating $\mathrm{G}_{1}$ progression and upregulating $G_{1}$ phase cyclin-dependent kinase 6 (CDK6), and improved cell survival by upregulating p38 in the MAPK cascade and increasing p-AKT in the AKT cascade, in particular in lung cancer cell lines. Overall, the results from the present study demonstrated that ENO1 may contribute to the development of lung cancers, but not esophageal cancers.

\section{Introduction}

Enolase (ENO), also known as 2-phospho-D-glycerate hydrolase, is a metalloenzyme that catalyzes the dehydration of 2-phospho-D-glycerate into phosphoenolpyruvate in the glycolytic pathway (1). There are three different ENO isoforms in higher eukaryotes, including ENO1 or $\alpha$-ENO, ENO2 or $\gamma$-ENO and ENO3 or $\beta$-ENO (2). Furthermore, the expression of the different ENO isoforms is tissue specificity. ENO1 is expressed in almost all kinds of tissue, whereas ENO3 is restricted to muscle tissues and ENO2 is described as a neuron- and neuroendocrine tissue-specific enolase (1).

ENO1 serves multifunctional roles in physiological and pathological processes $(1,3)$. For example, ENO1 is involved with various types of human cancer. ENO1 is upregulated in retinoblastoma (4) and is considered a diagnostic marker of hepatocellular carcinoma (5), pancreatic cancer (6), renal cell carcinoma $(7,8)$ and cholangiocarcinoma (9). In addition, abnormally upregulated ENO1 is associated with poor survival and prognosis in patients with mammary carcinoma (10). Furthermore, overexpression of ectopic ENO1 promotes tumor formation or enhances cell transformation in gastric cancer (11), lung cancer (12), pancreatic cancer (13), colorectal cancer (14) and glioma (15) and promotes chemoresistance in gastric (16) and breast (17) cancers. ENO1 has also been considered a therapeutic target in endometrial carcinoma (18). Conversely, ENO1 was reported to induce apoptosis (19) and cell differentiation (20) and to be downregulated in certain 
types of cancer, including lung cancer (21), suggesting opposite roles of ENO1 in cancer formation. To the best of our knowledge, only two studies demonstrated the association between ENO1 and lung cancer (21); however, contradictory observations have led to an ambiguous role of ENO1 in lung cancer formation.

The present study aimed to investigate the cell proliferation stimulating effect of ENO1 in various cancer cell lines and to determine the role of ENO1 in lung cancer formation in particular. The results of the present study demonstrated that overexpression of ENO1 stimulates cell proliferation and survival in lung cancers compared with esophageal cancers, by upregulating cyclin-dependent kinase 6 (CDK6) and protein kinase B (p38), thereby reflecting the tissue-type specific contribution of ENO1 to cancer formation.

\section{Materials and methods}

Chemicals, plasmids, antibodies, short hairpin (sh)RNAs and plasmids. Fetal bovine serum (FBS), Dulbecco's modified Eagle's medium (DMEM), penicillin, streptomycin, and Lipofectamine $^{\mathrm{TM}}$ were purchased from Gibco; Thermo Fisher Scientific, Inc. The characteristics of the primary antibodies used were as follows: Anti-green fluorescent protein (GFP) (B-2) (1:1,000; cat. no. sc-9996; Santa Cruz Biotechnology, Inc.), anti-enolase (C-19) (1:1,000; cat. no. sc-7455; Santa Cruz Biotechnology Inc.), anti- $\beta$-actin (C-2) $(1: 1,000$; cat.no. sc-8432; Santa Cruz Biotechnology,Inc.), anti- $\alpha$-tubulin (B-7) (1:1,000; cat. no. sc-5286; Santa Cruz Biotechnology, Inc.); phospho-AKT pathway sampler kit (1:500; cat. no 9916; Cell Signaling Technology, Inc.), MAPK family sampler kit (1:500; cat. no. 9926; Cell Signaling Technology, Inc.), phospho-MAPK family sampler kit (1:500; cat. no. 9910; Cell Signaling Technology, Inc.), CDK sampler kit (1:1,000; cat. no. 9868; Cell Signaling Technology, Inc.) and cyclin sampler kit (1:500; cat. no. 9869; Cell Signaling Technology, Inc.). The expression vectors for enhanced GFP (pEGFP) was purchased from Invitrogen; Thermo Fisher Scientific, Inc. All short hairpin (sh)RNAs packed in lentivirus were provided by Academia Sinica. The target sequences of shRNA for Luciferase and ENO1 were GCGGTTGCCAAGAGGTTC CAT and CGTGAACGAGAAGTCCTGCAA, respectively. EGFP-ENO1 was constructed by polymerase chain reaction (PCR) cloning using human full-length ENO1 cDNA clone purchased from the mammalian genome collection American Type Culture Collection (ATCC) as a template.

Patient samples and microarray analysis. The study protocol for the collection of patients' biopsies was approved by the Ethics Committee of the Taichung Veterans General Hospital. All the patients provided signed informed consent prior to the study. No patient had received neoadjuvant treatment prior to surgery. For lung cancer samples, 58 patients were recruited between April 2004 and December 2005 (76\% men; 24\% women; mean age, 69 years; age range, $49-86$ years) at the Taichung Veterans General Hospital. For esophageal cancer samples, eight patients were recruited between October 2003 and October 2005 (100\% men; mean age, 59 years; age range, 49-77 years). Samples were obtained following tumor resection from a non-necrotic area of the tumor and from adjacent non-tumorous tissues from neighboring sites. The tumorous and non-tumorous status of the two sample types was confirmed by pathologists. Tissue samples were immediately placed in cryovials, frozen in liquid nitrogen and stored at $-80^{\circ} \mathrm{C}$ until further analysis.

Cell culture, gene transfection and RNA interference. All the cell lines used in this study were purchased from ATCC and some were subjected to authentication. The HepG2 liver cancer cell line and TE12 esophageal cancer cell line were authenticated via STR DNA fingerprinting using the Promega GenePrint System (Promega Corporation) and ABI GeneMapper software (version 4.0; Applied Biosystems; Thermo Fisher Scientific, Inc.). The resulting STR profiles matched with ATCC fingerprints for HepG2 or ExPASy Database for TE12. The cell lines 293, 293T, HepG2 and HeLa were cultured in DMEM completed with 5\% FBS. A549, H1299, H460, and TE12 cell lines were cultured in RPMI-1640 containing $10 \%$ FBS, $1 \%$ non-essential amino acids and $1 \%$ sodium pyruvate (Gibco; Thermo Fisher Scientific, Inc.). Furthermore, $2 \mathrm{mM}$ glutamine, $100 \mathrm{U} / \mathrm{ml}$ penicillin and $100 \mu \mathrm{g} / \mathrm{ml}$ streptomycin were added in all cell media. Cells were placed at $37^{\circ} \mathrm{C}$ in a humidified incubator containing $5 \% \mathrm{CO}_{2}$. The respective cell lines (293T, 293, HepG2, HeLa, H1299, H460, A549 and TE12) were seeded into a $10-\mathrm{cm}$ dish at a density of 30,000 cells, respectively, and transfected with $4 \mu \mathrm{g}$ of EGFP empty vector or EGFP-ENO1 using Lipofectamine $2000^{\mathrm{TM}}$ according to the manufacturer's instructions. Western blotting and single cell proliferation were performed following 24 and $72 \mathrm{~h}$ transfection, respectively. Regarding RNA interference, the shRNAs packed in lentivirus and provided by the National RNAi core facility (Institute of Molecular Biology, Academia Sinica, Taiwan, R.O.C), were employed to infect cells in the presence of $8 \mu \mathrm{g} / \mathrm{ml}$ polybrene, which notably stimulates infection rate. The infected $\mathrm{H} 460$ and $\mathrm{H} 1299$ cells were subsequently positively selected with puromycin, and western blotting was performed to confirm the knockdown effect.

Single cell proliferation assay. This assay was performed as previously described (22). Briefly, the cells (293T, 293, HepG2, HeLa, A549, TE12, H1299 and H460) were seeded in a 6-cm dish until they reached $50 \%$ confluence and subsequently transfected with constructs tagged with EGFP. After $24 \mathrm{~h}$, 30,000 cells were reseeded into a $10-\mathrm{cm}$ dish, so that each cell remained single and could not make contact with each other cells. Cells were allowed to proliferate for $48 \mathrm{~h}$, and the number of cells that formed 'mini-colony' of $\geq 2$ cells was counted using a fluorescence microscope (Olympus Corporation; magnification, $\mathrm{x} 200$ ). Cells that did not proliferate remained single in the dish after 2 days of culture.

Flow cytometry. Cells were trypsinized, washed with PBS and fixed in $70 \%$ ethanol at $-20^{\circ} \mathrm{C}$ for $20 \mathrm{~min}$. Cells were washed with PBS, incubated with $100 \mu \mathrm{g} / \mathrm{ml}$ RNase (Sigma-Aldrich; Merck $\mathrm{KGaA}$ ) at $37^{\circ} \mathrm{C}$ for $30 \mathrm{~min}$, stained with propidium iodide $(50 \mu \mathrm{g} / \mathrm{ml}$; Sigma-Aldrich; Merck KGaA) at room temperature for $1 \mathrm{~h}$, and analyzed on a FACScan ${ }^{\mathrm{TM}}$ flow cytometer (BD Biosciences). The percentage of each cell cycle phase was analyzed using Cell-FIT software and BD FACSDiva ${ }^{\mathrm{TM}}$ software (version 8.0.1; BD Diagnostics). 
A
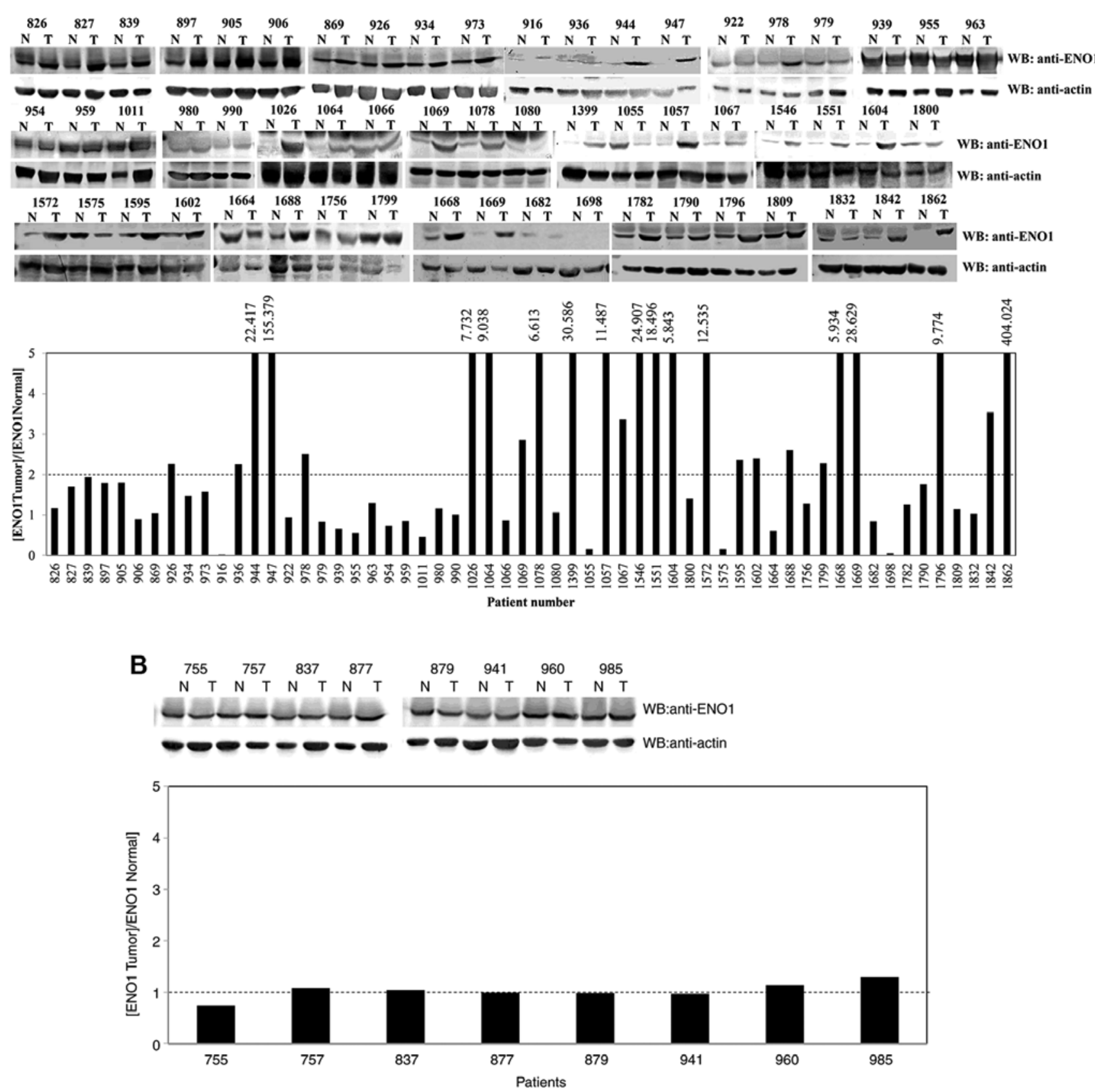

Figure 1. ENO1 was overexpressed in lung cancer tissues. (A) Expression pattern of ENO1 in a collected panel of lung cancer tissues. Protein expression of ENO1 was analyzed by western blotting in 58 lung cancer tissues. Expression of ENO1 and $\beta$-actin was analyzed by western blotting in biopsies from paired lung tumor and adjacent normal tissues. The numbers above the western blotting images or on the $\mathrm{x}$ axis of the quantification graph represent the codes of patients. The ratio of normalized tumor ENO1 to normal tissue ENO1 was calculated and plotted on a graph. (B) Expression pattern of ENO1 in a collected panel of esophageal cancer tissues. ENO1 protein expression was determined by western blotting in eight esophageal cancer tissue samples. Expression of ENO1 was analyzed by western blotting in biopsies from paired esophageal tumor and adjacent normal tissues. The numbers above the western blotting images or on the $\mathrm{x}$ axis of the quantification graph represent the codes of patients. The intensity of ENO1 or $\beta$-actin was quantified via densitometry. The level of ENO1 protein in each paired tissue was normalized against that of $\beta$-actin. The ratio of normalized tumor ENO1 to normal tissue ENO1 was calculated and plotted on a graph. ENO1, enolase 1; $\mathrm{N}$, normal adjacent tissue; $\mathrm{T}$, tumor tissue.

Protein samples preparation and western blotting. Cells were lysed with extraction buffer containing $20 \mathrm{mM}$ PIPES (pH 7.2), $100 \mathrm{mM} \mathrm{NaCl}, 1 \mathrm{mM}$ EDTA, $0.1 \%$ CHAPS, $10 \%$ sucrose, $1 \mathrm{mM}$ PMSF, $1 \mathrm{mM}$ DTT, $1 \mathrm{mM} \mathrm{Na} \mathrm{VO}_{4}$, and $10 \mu \mathrm{g} / \mathrm{ml}$ of leupeptin, aprotinin, chymostatin and pepstatin (Thermo Fisher Scientific, Inc.) at $4^{\circ} \mathrm{C}$ for $30 \mathrm{~min}$. Cellular debris was removed by centrifugation at $15,700 \mathrm{x}$ g for $90 \mathrm{~min}$ at $4^{\circ} \mathrm{C}$ in an Eppendorf centrifuge. Protein concentration was determined using Bradford assay (Bio-Rad Laboratories,
Inc.). The total amount of protein extracts loaded for the detection of phosphorylated protein, total protein, and for internal control and exogenous transfected protein was 200 , 100 and $50 \mu \mathrm{g}$, respectively. The resulting samples were heated at $95^{\circ} \mathrm{C}$ for $10 \mathrm{~min}$, separated by $10 \%$ SDS-PAGE and transferred onto polyvinylidene difluoride membrane (EMD Millipore). Membrane was blocked with 5\% bovine serum albumin diluted in PBS containing 0.1\% Tween-20 (PBST) at room temperature for $1 \mathrm{~h}$. Membranes were incubated with 
A

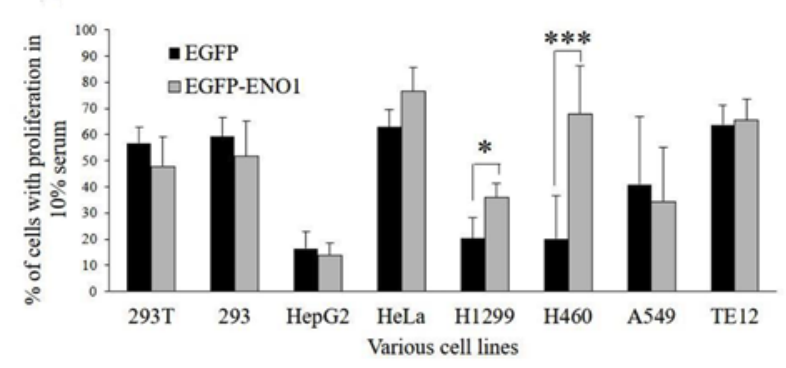

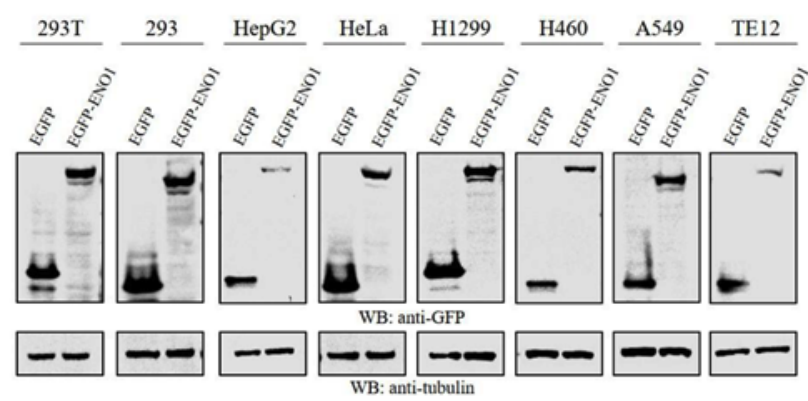

B

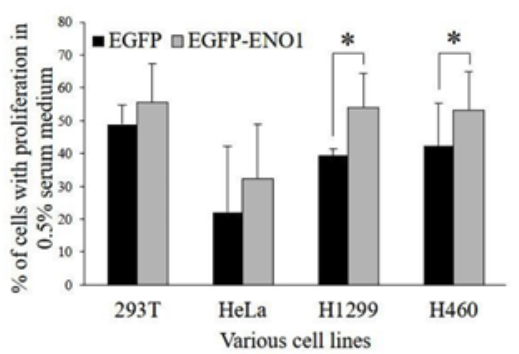

C

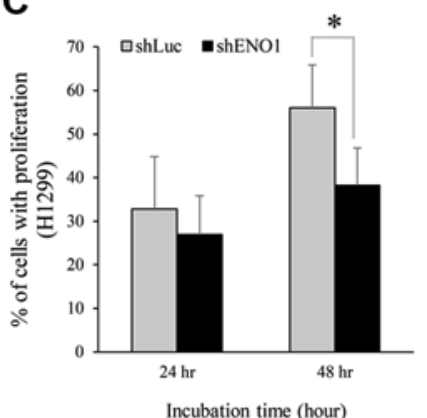

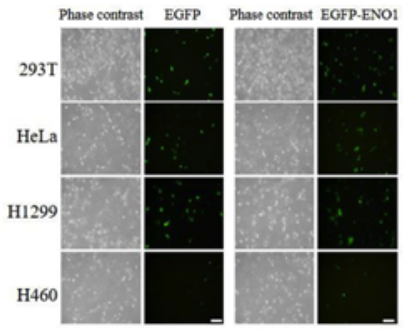

D

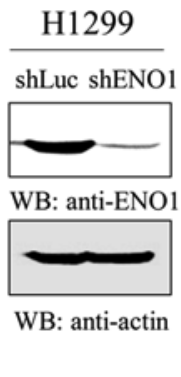

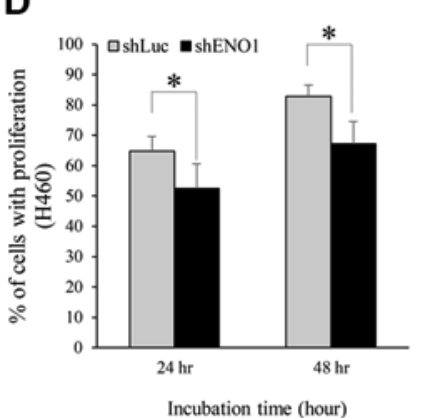

H460

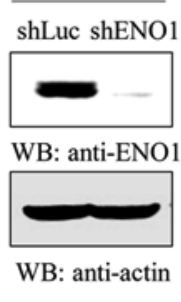

Figure 2. ENO1 selectively stimulated H1299 and H460 cell proliferation or survival in normal and low serum medium. (A and B) Various cell lines transfected with EGFP or EGFP-ENO1 were subjected to single cell proliferation for $48 \mathrm{~h}$ in medium containing (A) $10.0 \%$ or (B) $0.5 \%$ fetal bovine serum. The percentage of cell proliferation was counted and plotted. The protein expression of exogenous EGFP-ENO1 and images of cells harboring EGFP or EGFP-ENO1 were displayed on the right of (A) and (B) respectively. Scale bar, $2 \mathrm{~mm}$; magnification, x200. (C and D) ENO1 knockdown reduced H1299 and H460 cell proliferation. (C) H1299 or (D) H460 cells harboring shLuc or shENO1 were analyzed for single cell proliferation. ${ }^{*} \mathrm{P}<0.05$ and ${ }^{* * * *} \mathrm{P}<0.001$. EGFP, enhanced green fluorescent protein; ENO1, enolase 1; Luc, luciferase; sh, short hairpin; WB, western blotting.

primary antibodies at $4^{\circ} \mathrm{C}$ overnight. Membranes were washed three times with PBST at room temperature for $30 \mathrm{~min}$ and incubated with horseradish peroxidase (HRP)-conjugated anti-rabbit (cat. no. sc2004) or anti-mouse (cat. no. sc2005) immunoglobulin G secondary antibodies (both 1:10,000 and from Santa Cruz Biotechnology, Inc.) for $1 \mathrm{~h}$ at room temperature. Membranes were then washed three times with PBST for $30 \mathrm{~min}$ at room temperature. HRP substrates (cat. no. 32106; Thermo Fisher Scientific, Inc.) were used to detect the signal on the membrane and protein expression was quantified using Gel Pro Analyzer software (version 4.0; Media Cybernetics, Inc).

Statistical analysis. Statistical analysis was performed using Excel 2016. Data were collected from three independent experiments and are expressed as the mean \pm standard deviation. Comparison of cell proliferation between EGFPand EGFP-ENO1-transfected cells, or between shLuc- and shENO1-transfected cells was performed using a paired Student's t-test. $\mathrm{P}<0.05$ was considered to indicate a statistically significant difference.

\section{Results}

ENO1 is abnormally upregulated in lung cancer tissues, but not in esophageal cancer tissues. The present study determined the protein expression level of ENO1 in 58 lung paired tissues and eight esophageal paired tissues (cancerous and adjacent normal biopsies). The results demonstrated that 25 lung cancer paired tissues expressed a 2-fold higher level of ENO1 in the cancerous tissues compared with the normal tissues (Fig. 1A). Similar protein levels of ENO1 were observed in the cancerous and normal biopsies of all esophageal paired tissues (Fig. 1B). These results suggested that ENO1 may serve a role in the development of lung cancer.

ENO1 is essential for lung cancer but not esophageal cancer cell proliferation. In order to investigate the effects of ENO1 on cell proliferation, EGFP or EGFP-ENO1 were transfected in various cell lines cultured in medium containing $10 \%$ (Fig. 2A) or $0.5 \%$ (Fig. 2B) FBS, including kidney epithelium (293T and 293), liver cancer (HepG2), cervical cancer (HeLa), lung cancer (H1299, H460 and A549) and esophageal cancer 
A
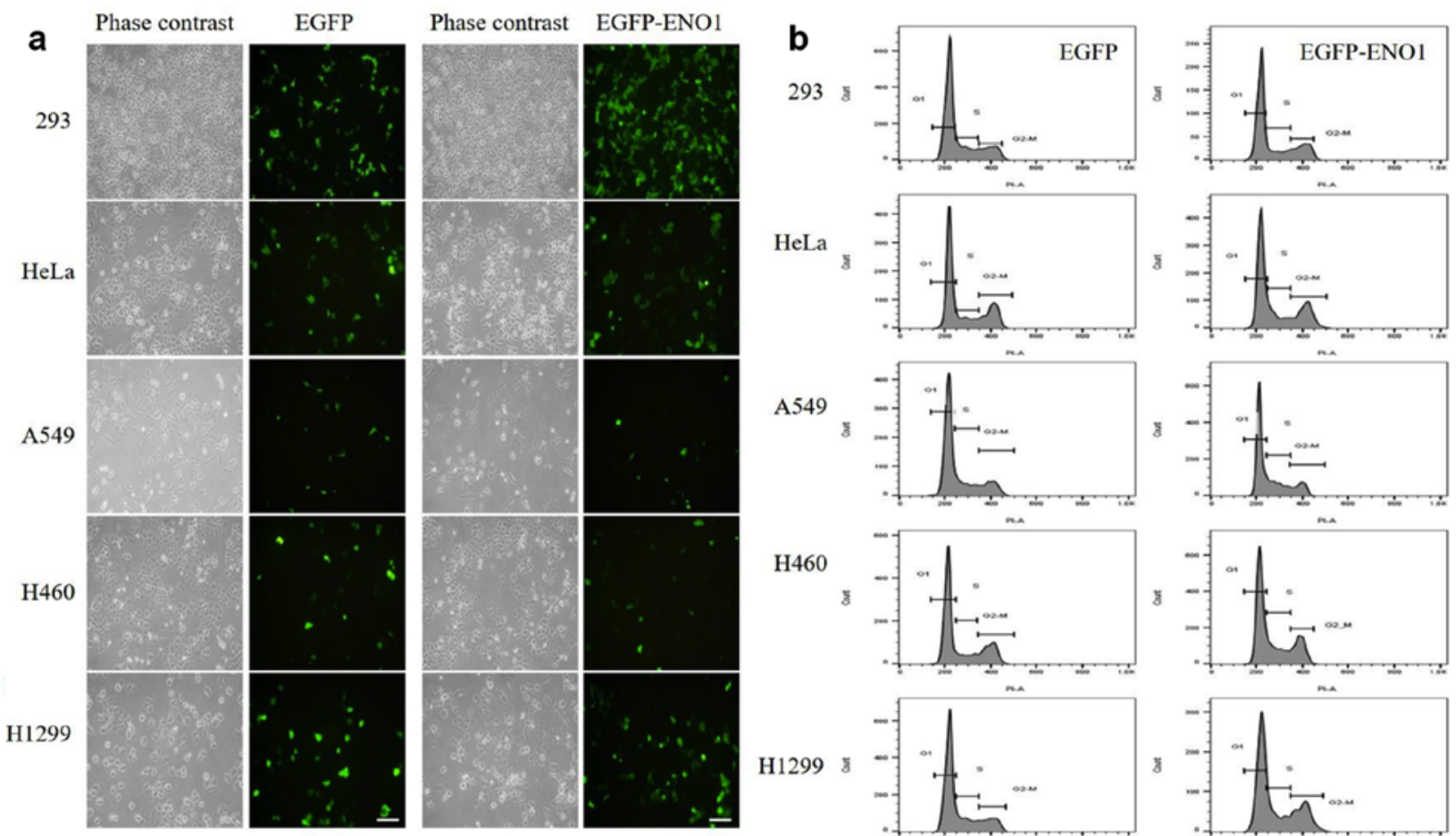

H460
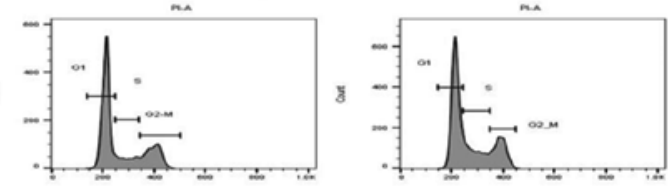

C

293
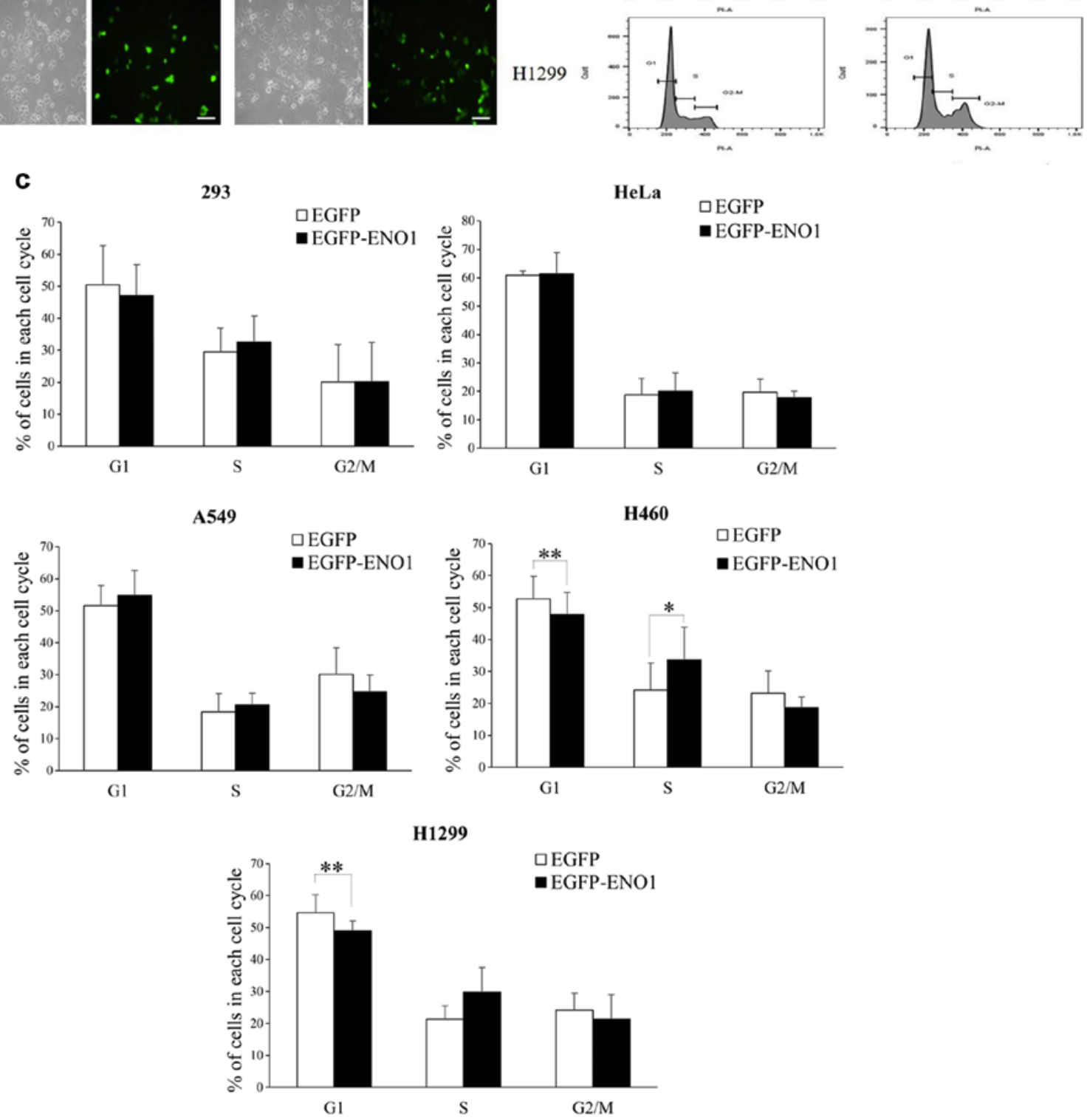

Figure 3. ENO1 stimulated cell proliferation by accelerating $\mathrm{G}_{1}$ progression and upregulating CDK6 in H1299 cells. (A) ENO1 accelerated $\mathrm{G}_{1}$ progression. Flow cytometric analysis was performed in various cell lines harboring EGFP or EGFP-ENO1. (Aa and Ab) Cells harboring EGFP or EGFP-ENO1 were chosen for DNA content analysis by flow cytometer, and (Ac) the \% of cells in $\mathrm{G}_{1}\left(2 \mathrm{X}\right.$ DNA), $\mathrm{S}\left(2 \mathrm{X}-4 \mathrm{X}\right.$ DNA) and $\mathrm{G}_{2} / \mathrm{M}$ (4X DNA) phases was calculated and plotted. Scale bar, $2 \mathrm{~mm}$; magnification, $\mathrm{x} 200 .{ }^{*} \mathrm{P}<0.05 ;{ }^{* *} \mathrm{P}<0.01$. CDK, cyclin-dependent kinase. 

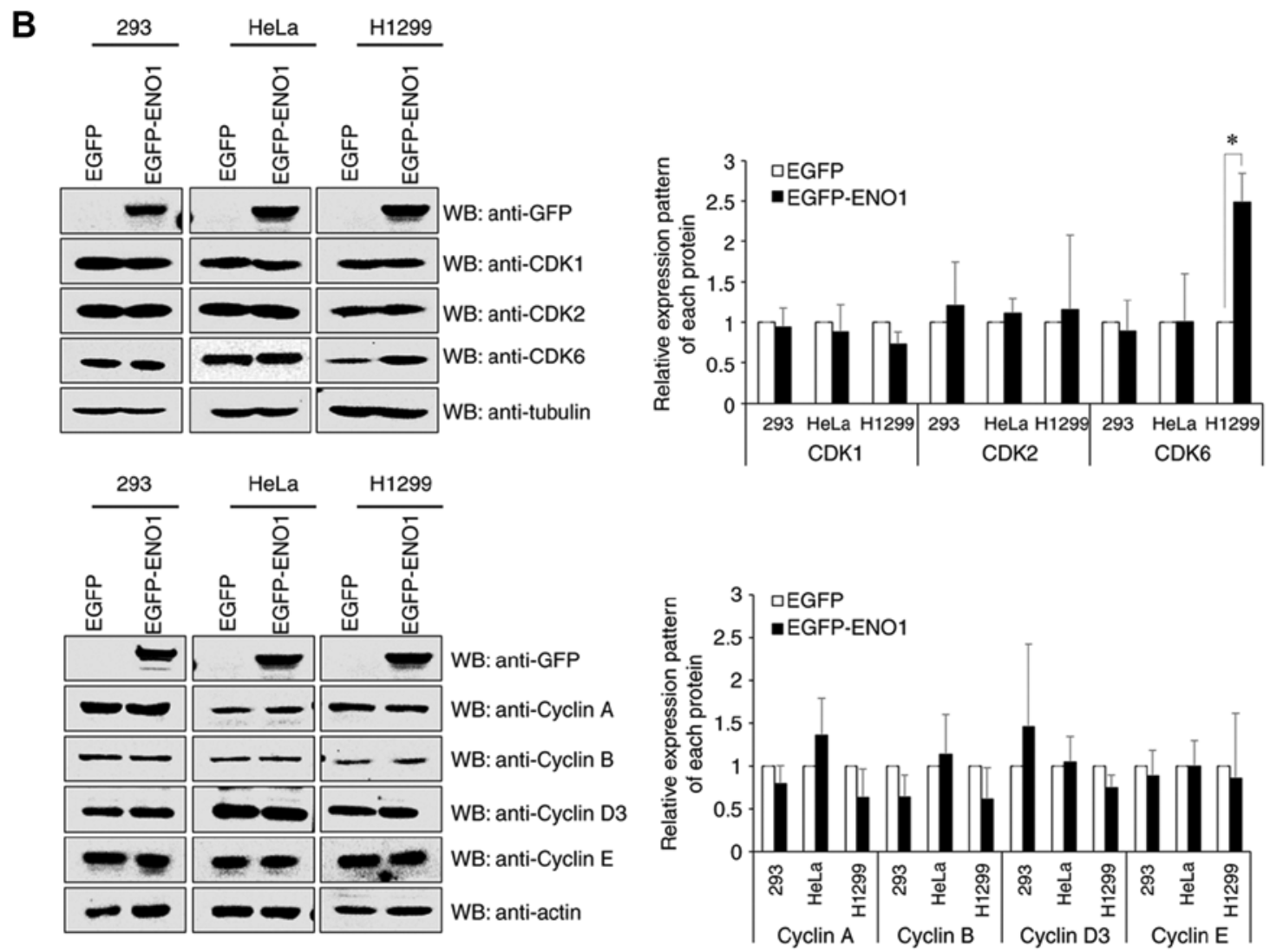

Figure 3. Continued. (B) ENO1 upregulated CDK6 in H1299 cells. Western blotting for the protein expression of CDKs (upper) and cyclins (lower) was performed in EGFP or EGFP-ENO1-transfected cells. The relative expression of each protein was measured and plotted on the right. ${ }^{*} \mathrm{P}<0.05$. CDK, cyclin-dependent kinase; WB, western blotting.

(TE12). The results demonstrated that overexpression of ectopic ENO1 stimulated the proliferation of two lung cancer cell lines H1299 and H460, but had no effect on other cell line proliferation, including the esophageal cell line TE12. Consistently with the results from overexpression, ENO1 knockdown inhibited cell proliferation in H1299 and H460 cell proliferation (Fig. 2C and D).

It has been reported that the transcript of ENO1 is elevated in lung cancer tissues, and that ENO1 stable cell lines grow into colonies, migrate faster and form tumors in mice (12). The results from the present study indicated that there is a high prevalence of increased ENO1 protein level in lung cancer tissues. Furthermore, ENO1 overexpression enhanced cell proliferation and cell survival in medium containing low serum (Fig. 2B). These results suggest that ENO1 may contribute to lung cancer cell transformation, although ENO1 downregulation has also been reported in lung cancer (21).

ENO1 stimulates cell proliferation by accelerating $G_{I} / S$ transition. In order to investigate how ENO1 could stimulate cell proliferation, the impact of exogenous ENO1 on cell cycle progression was investigated. The results revealed that ENO1 accelerated $\mathrm{G}_{1}$ progression in $\mathrm{H} 460$ and $\mathrm{H} 1299$ cells but did not alter cell cycle in other cell lines (Fig. 3A). Further mechanistic analyses demonstrated that ENO1 upregulated the $\mathrm{G}_{1}$ CDK known as CDK6, in H1299 cell line, but not in 293 and HeLa cell lines (Fig. 3B). These results indicated that
ENO1 may promote H1299 proliferation via CDK6-mediated acceleration of $\mathrm{G}_{1}$. Furthermore, overexpression of exogenous ENO1 promoted cell proliferation and enhanced cell survival in lung cancer cell lines, but not in esophageal cell lines.

ENO1 upregulates p38 and activates AKT in H1299 cell line. In order to determine why ENO1 stimulated cell survival in $0.5 \%$ FBS (Fig. 2B), the two survival pathways MAPK (23) and AKT (24) were investigated. The results demonstrated that exogenous ENO1 induced the increase in p38 and p-AKT protein levels and the decrease in cell growth suppressive p-c-Raf protein level in H1299 cells (Fig. 4A), but not in A549 and 293T cell lines (Fig. 4B). These results suggested that ENO1 may promote cell survival by activating p38 and AKT.

\section{Discussion}

The results from the present study indicated that ENO1 was overexpressed in 25 out of $58(\sim 43 \%)$ lung cancer tissues, with ENO1 protein level 2-times higher in cancer tissues compared with normal tissues. Furthermore, ENO1 protein level was 5-fold higher in 15 lung cancer tissues ( 26\%), which indicated that ENO1 was commonly upregulated in lung cancer tissues. However, due to the insufficient amount of tissue biopsies, these results could not be confirmed by immunohistochemical analysis. Conversely, ENO1 was not overexpressed in the eight esophageal cancer samples, which 
A

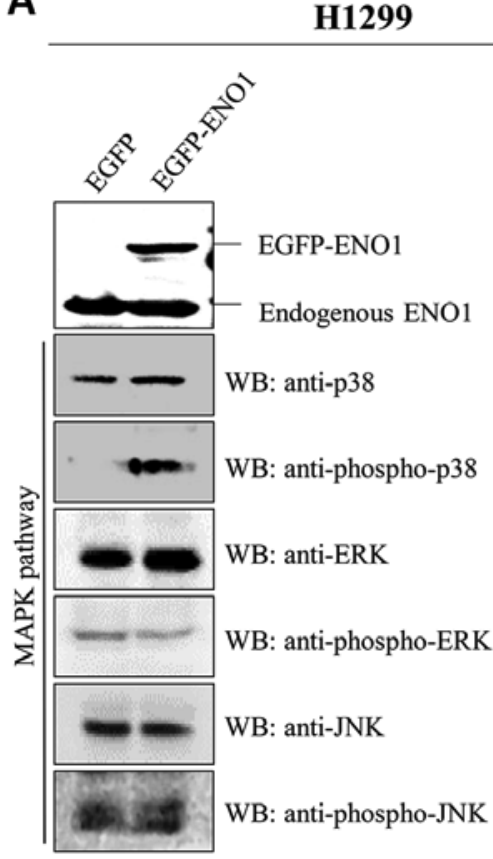

B

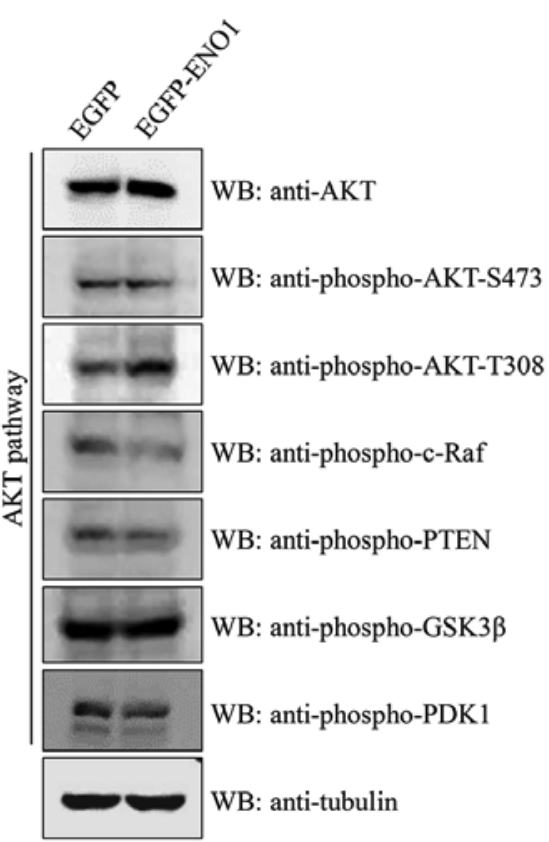

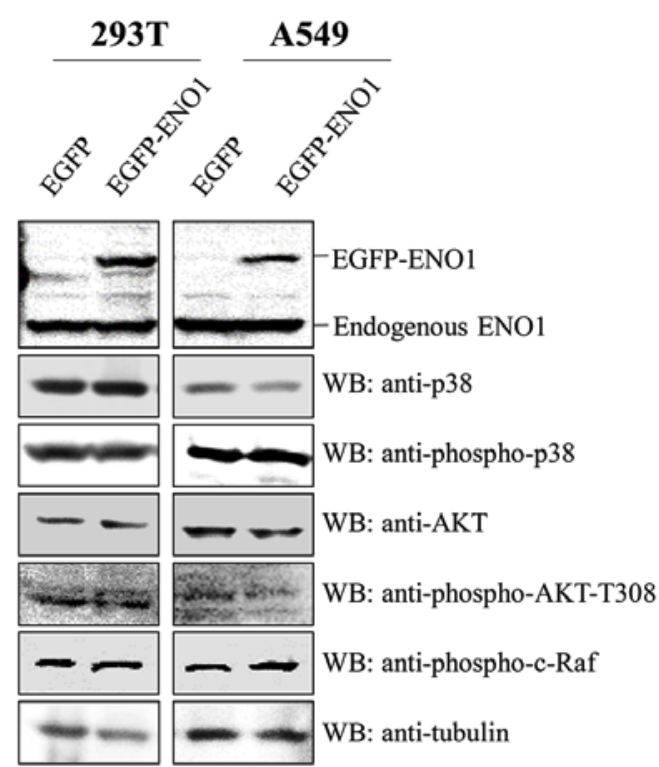

Figure 4. ENO1 overexpression induced the increase of p38, p-AKT and p-c-Raf protein levels in H1299 cells. (A) Effects of ectopic ENO1 on MAPK and AKT pathways in H1299 cells. Proteins levels from the MAPK and AKT cascades were analyzed by western blotting in EGFP and EGFP-ENO1-transfected cells. (B) Effects of ectopic ENO1 on the MAPK and AKT pathways in 293T and A549 cells. Proteins levels from the MAPK and AKT cascades were analyzed by western blotting in EGFP and EGFP-ENO1-transfected cells. EGFP, enhanced green fluorescent protein; ENO1, enolase 1; GSK3 $\beta$, Glycogen synthase kinase 3 $\beta$; PDK1, pyruvate dehydrogenase kinase 1; PTEN, phosphatase and tensin homolog; WB, western blotting.

revealed the distinctive regulation of ENO1 in the two cancer types. In addition, consistent with the aforementioned results, exogenous ENO1 preferentially stimulated cell proliferation of the lung cancer cell lines H1299 and H460, but not of the esophageal TE12, liver cancer HepG2 and cervical cancer HeLa cell lines.

Mechanistic analyses revealed that ENO1 overexpression upregulated CDK6 and p38 protein levels, and suppressed c-Raf in H1299 cells compared to other cell lines. Furthermore, ENO1 overexpression only activated AKT to a certain extent, as full activation of AKT requires phosphorylation at T308 and T473 (25), and only stimulated the increase of AKT-thr308. In addition, ectopic ENO1 accelerated $G_{1}$ progression and upregulated CDK6, rather than $\mathrm{G}_{1}$ cyclins, including cyclins A, B, D or E. Furthermore, the growth suppressor protein c-Raf was decreased following ENO1 overexpression. These results, along with analysis at low serum levels, suggest that ENO1 may enhance cell proliferation and cell transformation primarily by upregulating CDK6 and p38 expression and downregulating c-Raf expression in $\mathrm{H} 1299$ cell line, but not in 293T or A549 cell lines.

ENO1 has been reported to activate AKT in gastric cancer, pulmonary artery cancer and primary non-small cell lung cancer tissues via the PI3K, AMP-activated protein kinase and focal adhesion kinase (FAK) pathways, respectively $(26,11,12)$. Since H1299 and H460 are non-small lung cancer cell lines, ENO1 may therefore activate AKT via the FAK pathway. To the best of our knowledge there is currently no evidence to support the association between ENO1 and p38, although it has been reported that ENO1 may serve as a receptor for plasminogen (27), plasmin being able to activate p38 (28).
It is well established that ENO1 can stimulate cell transformation or proliferation via its glycolytic activity (29). The results of the present study, which demonstrated that ENO1 failed to promote the proliferation of the lung cancer cell line A549, were inconsistent with the study from $\mathrm{Fu}$ et al (12), which demonstrated that ENO1 can enhance cell proliferation, colony formation and cell migration in A549 cell line. This could be due to the fact that the present study investigated the effects of ENO1 on cell proliferation via transient expression, whereas Fu et al used a stable cell line to examine the effect of ENO1 on A549 cell proliferation. Long-term incubation of A549 cells with exogenous ENO1 may offer additional advantages to lung cancer cell proliferation.

\section{Acknowledgements}

The authors of the present study would like to thank Ms Jia-Rong Tsai from the Division of Hematology and Oncology, Ms Mei-Chun Liu from the Instrument Center, and Ms Jen Miao and Ms Li-Wen Lee from the Division of Thoracic Surgery of the Taichung Veterans General Hospital for their technical support.

\section{Funding}

The present study was funded by the Taichung Veterans General Hospital-National Chi Nan University Joint Research Program (grant nos. TCVGH-NCNU1077902 and TCVGHNCNU1067902), the Taichung Veterans General Hospital (grant nos. TCVGH-1073208C and TCVGH-1073210D), the Ministry of Science and Technology (grant no. MOST 
105-2320-B-260-001-MY3) and the China Medical University and Hospital grant (grant no. DMR-107-116).

\section{Availability of data and materials}

The datasets used and/or analyzed during the current study are available from the corresponding author on reasonable request.

\section{Authors' contributions}

CY, SC and KC designed the present study, performed the literature review, and analyzed and interpreted the data. CY drafted the initial manuscript. JC performed most of the experiments. YH and YL performed western blotting. All authors read and approved the final manuscript.

\section{Ethics approval and consent to participate}

The present study was approved by The Institutional Review Board and the Human Ethics Committee of Taichung Veterans General Hospital (Taichung, Taiwan). Written informed consent was obtained from all patients prior to the study start.

\section{Patient consent for publication}

Not applicable.

\section{Competing interests}

The authors declare that they have no competing interests.

\section{References}

1. Pancholi V: Multifunctional alpha-enolase: Its role in diseases. Cell Mol Life Sci 58: 902-920, 2001.

2. Verma $M$ and Dutta SK: DNA sequences encoding enolase are remarkably conserved from yeast to mammals. Life Sci 55: 893-899, 1994

3. Díaz-Ramos A, Roig-Borrellas A, García-Melero A and López-Alemany R: $\alpha$-Enolase, a multifunctional protein: Its role on pathophysiological situations. Nd J Biomed Biotechnol 2012: $156795,2012$.

4. Nakajima T, Kato K, Kaneko A, Tsumuraya M, Morinaga S and Shimosato Y: High concentrations of enolase, alpha- and gamma-subunits, in the aqueous humor in cases of retinoblastoma. Am J Ophthalmol 101: 102-106, 1986.

5. Zhu W,LiH, Yu Y, Chen J, Chen X,Ren F, Ren Z and Cui G: Enolase-1 serves as a biomarker of diagnosis and prognosis in hepatocellular carcinoma patients. Cancer Manag Res 10: 5735-5745, 2018.

6. Sun L, Guo C, Cao J, Burnett J, Yang Z, Ran Y and Sun D: Over-expression of alpha-enolase as a prognostic biomarker in patients with pancreatic cancer. Int J Med Sci 14: 655-661, 2017.

7. Kaneko N, Gotoh A, Okamura N, Matsuo E, Terao S, Watanabe M, Yamada Y, Hamami G, Nakamura T, Ikekita M, et al: Potential tumor markers of renal cell carcinoma: A-enolase for postoperative follow up, and galectin-1 and galectin-3 for primary detection. Int J Urol 20: 530-535, 2013.

8. White-AI Habeeb NM, Di Meo A, Scorilas A, Rotondo F, Masui O, Seivwright A, Gabril M, Girgis AH, Jewett MA and Yousef GM: Alpha-enolase is a potential prognostic marker in clear cell renal cell carcinoma. Clin Exp Metastasis 32: 531-541, 2015.

9. Yonglitthipagon P, Pairojkul C, Bhudhisawasdi V, Mulvenna J, Loukas A and Sripa B: Proteomics-based identification of $\alpha$-enolase as a potential prognostic marker in cholangiocarcinoma. Clin Biochem 45: 827-834, 2012.

10. Chu PY, Hsu NC, Liao AT, Shih NY, Hou MF and Liu CH: Overexpression of $\alpha$-enolase correlates with poor survival in canine mammary carcinoma. BMC Vet Res 7: 62, 2011.
11. Sun L, Lu T, Tian K, Zhou D, Yuan J, Wang X, Zhu Z, Wan D, Yao Y, Zhu X and He S: Alpha-enolase promotes gastric cancer cell proliferation and metastasis via regulating AKT signaling pathway. Eur J Pharmacol 845: 8-15, 2018.

12. Fu QF, Liu Y, Fan Y, Hua SN, Qu HY, Dong SW, Li RL, Zhao MY, Zhen Y, Yu XL, et al: Alpha-enolase promotes cell glycolysis, growth, migration, and invasion in non-small cell lung cancer through FAK-mediated PI3K/AKT pathway. J Hematol Oncol 8: $22,2015$.

13. Principe $\mathrm{M}$, Borgoni S, Cascione M, Chattaragada MS Ferri-Borgogno S, Capello M, Bulfamante S, Chapelle J, Di Modugno F, Defilippi P, et al: Alpha-enolase (ENO1) controls alpha $\mathrm{v} /$ beta 3 integrin expression and regulates pancreatic cancer adhesion, invasion, and metastasis. J Hematol Oncol 10: 16, 2017.

14. Zhan P, Zhao S, Yan H, Yin C, Xiao Y, Wang Y, Ni R, Chen W, Wei $\mathrm{G}$ and Zhang P: $\alpha$-enolase promotes tumorigenesis and metastasis via regulating AMPK/mTOR pathway in colorectal cancer. Mol Carcinog 56: 1427-1437, 2017.

15. Song Y, Luo Q, Long H, Hu Z, Que T, Zhang X, Li Z, Wang G, Yi L, Liu Z, et al: Alpha-enolase as a potential cancer prognostic marker promotes cell growth, migration, and invasion in glioma. Mol Cancer 13: 65, 2014.

16. Qian X, Xu W, Xu J, Shi Q, Li J, Weng Y, Jiang Z, Feng L, Wang $X$, Zhou $J$ and Jin H: Enolase 1 stimulates glycolysis to promote chemoresistance in gastric cancer. Oncotarget 8: 47691-47708, 2017.

17. Tu SH, Chang CC, Chen CS, Tam KW, Wang YJ, Lee CH, Lin HW, Cheng TC, Huang CS, Chu JS, et al: Increased expression of enolase alpha in human breast cancer confers tamoxifen resistance in human breast cancer cells. Breast Cancer Res Treat 121: 539-553, 2010.

18. Zhao M, Fang W, Wang Y, Guo S, Shu L, Wang L, Chen Y, Fu Q, Liu Y, Hua S, et al: Enolase-1 is a therapeutic target in endometrial carcinoma. Oncotarget 6: 15610-15627, 2015.

19. Gao S, Li H, Feng XJ, Li M, Liu ZP, Cai Y, Lu J, Huang XY, Wang JJ, Li Q, et al: $\alpha$-Enolase plays a catalytically independent role in doxorubicin-induced cardiomyocyte apoptosis and mitochondrial dysfunction. J Mol Cell Cardiol 79: 92-103, 2015.

20. Lopez-Alemany R, Suelves M, Diaz-Ramos A, Vidal B and Munoz-Canoves P: Alpha-enolase plasminogen receptor in myogenesis. Front Biosci 10: 30-36, 2005.

21. Chang YS, Wu W, Walsh G, Hong WK and Mao L: Enolase-alpha is frequently down-regulated in non-small cell lung cancer and predicts aggressive biological behavior. Clin Cancer Res 9: 3641-3644, 2003.

22. Yu CT, Hsia JY, Hseih YC, Su LJ, Lee TC, Ku CF, Chen KS, Chen JM, Wei TY, Lee YC, et al: The novel protein suppressed in lung cancer down-regulated in lung cancer tissues retards cell proliferation and inhibits the oncokinase Aurora-A. J Thorac Oncol 6: 988-997, 2011.

23. Bonni A, Brunet A, West AE, Datta SR, Takasu MA and Greenberg ME: Cell survival promoted by the Ras-MAPK signaling pathway by transcription-dependent and -independent mechanisms. Science 286: 1358-1362, 1999.

24. Song G, Ouyang G and Bao S: The activation of Akt/PKB signaling pathway and cell survival. J Cell Mol Med 9: 59-71, 2005.

25. Facchinetti V, Ouyang W, Wei H, Soto N, Lazorchak A, Gould C, Lowry C, Newton AC, Mao Y, Miao RQ, et al: The mammalian target of rapamycin complex 2 controls folding and stability of Akt and protein kinase C. EMBO J 27: 1932-1943, 2008.

26. Dai J, Zhou Q, Chen J, Rexius-Hall ML, Rehman J and Zhou G: Alpha-enolase regulates the malignant phenotype of pulmonary artery smooth muscle cells via the AMPK-Akt pathway. Nat Commun 9: 3850, 2018.

27. Wygrecka M, Marsh LM, Morty RE, Henneke I, Guenther A, Lohmeyer J, Markart P and Preissner KT: Enolase-1 promotes plasminogen-mediated recruitment of monocytes to the acutely inflamed lung. Blood 113: 5588-5598, 2009.

28. Burysek L, Syrovets T and Simmet T: The serine protease plasmin triggers expression of MCP-1 and CD40 in human primary monocytes via activation of $\mathrm{p} 38$ MAPK and janus kinase (JAK)/STAT signaling pathways. J Biol Chem 277: 33509-33517, 2002.

29. Ji H, Wang J, Guo J, Li Y, Lian S, Guo W, Yang H, Kong F, Zhen L, Guo L and Liu Y: Progress in the biological function of alpha-enolase. Anim Nutr 2: 12-17, 2016.

This work is licensed under a Creative Commons Attribution-NonCommercial-NoDerivatives 4.0 International (CC BY-NC-ND 4.0) License. 